\title{
Structures of Zymomonas 2-Keto-3-Deoxy-6-Phosphogluconate Aldolase with and without a Substrate Analog at the Phosphate-Binding Loop
}

\author{
Pil-Won Seo ${ }^{1}$, Ho-Chang Ryu ${ }^{1}$, Do-Heon Gu ${ }^{1}$, Hee-Sae Park ${ }^{2}$, Suk-Youl Park ${ }^{3}$, and Jeong-Sun Kim ${ }^{1 *}$ \\ ${ }^{1}$ Department of Chemistry, Chonnam National University, Gwangju 61186, Republic of Korea \\ ${ }^{2}$ School of Biological Sciences and Technology, Hormone Research Center, Chonnam National University, Gwangju 61186, Republic of Korea \\ ${ }^{3}$ Pohang Accelerator Laboratory, Pohang, Gyeongbuk 37673, Republic of Korea
}

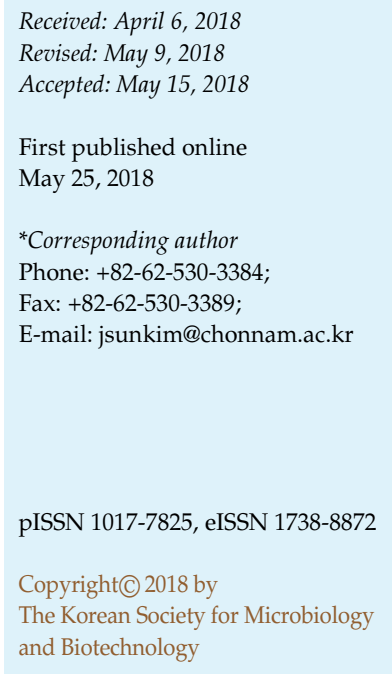

\begin{abstract}
2-Keto-3-deoxy-6-phosphogluconate (KDPG) aldolase, which catalyzes aldol cleavage and condensation reactions, has two distinct substrate-binding sites. The substrate-binding mode at the catalytic site and Schiff-base formation have been well studied. However, structural information on the phosphate-binding loop (P-loop) is limited. Zymomonas mobilis KDPG aldolase is one of the aldolases with a wide substrate spectrum. Its structure in complex with the substrate-mimicking 3-phosphoglycerate (3PG) shows that the phosphate moiety of 3PG interacts with the P-loop and a nearby conserved serine residue. 3PG-binding to the P-loop replaces water molecules aligned from the P-loop to the catalytic site, as observed in the apostructure. The extra electron density near the P-loop and comparison with other aldolases suggest the diversity and flexibility of the serine-containing loop among KDPG aldolases. These structural data may help to understand the substrate-binding mode and the broad substrate specificity of the Zymomonas KDPG aldolase.
\end{abstract}

Keywords: 2-Keto-3-deoxy-6-phosphogluconate aldolase (KDPG), aldolase, P-loop

\section{Introduction}

Many organisms use the Embden-Meyerhof-Parnas (EMP) pathway to get energy. In this pathway, glucose is successively phosphorylated and isomerized into fructose1,6-bisphosphate, which is further degraded into a dihydroxyacetone phosphate and a D-glyceraldehyde-3phosphate (GAP) [1]. On the other hand, some organisms, including the ethanologenic bacterium Zymomonas mobilis ZM4 [2], lack enzymes for the second phosphorylation and the isomerization of a hexose sugar derivative that are necessary in the EMP pathway. Instead, these organisms have an Entner-Doudoroff pathway and can covert the glucose into two 3-carbon molecules using 2-keto-3-deoxy6-phosphogluconate (KDPG) aldolase [1].

KDPG aldolases (E.C. 4.1.2.14) catalyze the cleavage reaction of KDPG into pyruvate and GAP [3]. They can also catalyze the reversible condensation reaction of the two triose phosphates of pyruvate and GAP into a hexose phosphate. Aldolases can be divided into two types depending on the catalytic mechanism: class I enzymes form a Schiff-base intermediate using the terminal nitrogen atom of the lysine side chain with the carbonyl moiety of the substrate [4-6], whereas class II enzymes, without the formation of a Schiff-base intermediate, catalyze the reaction aided by a metal cofactor [7-10]. Since aldolases can mediate a chemical reaction to introduce a covalent bond between two carbon atoms, many living organisms utilize aldolases for the biosynthesis of a large carbon skeleton. Enzyme-catalyzed reactions give products with a high stereoselectivity. Therefore, aldolases have also been the subject of a particular interest in organic synthesis, including the manufacture of nikkomycin analogs [11].

Bacterial class I KDPG aldolases are proteins composed of 200 amino acids. Crystal structures of KDPG aldolases from five different microorganisms have revealed a common homotrimeric structure in the crystalline state [4, 5, 12-14]. Each protomer forms a TIM barrel structure with a central $\beta$-barrel of eight $\beta$-strands surrounded by the same number of $\alpha$-helices. In the Escherichia coli KDPG 
aldolase structure in complex with pyruvate [4], the catalytic lysine residue forms a Schiff-base with the substrate pyruvate, and the carboxylate moiety of pyruvate simultaneously interacts with a glutamate residue. In this structure, an aromatic phenylalanine residue close to the catalytic lysine has been suggested to confer stereospecificity on the C2 atom of the bound substrate. The inorganic sulfate or phosphateion at the phosphate-binding loop (Ploop) in the Escherichia coli [5] and the Thermotoga maritima [12] KDPG structures implies that the phosphate moiety attached to the C6 atom of KDPG interacts both with the two peptidyl amide nitrogen atoms of the P-loop and a hydroxyl group of the conserved serine residue. A recently suggested enzymatic mechanism of KDPG aldolases involves two catalytic water molecules that relay protons during the reaction [12]. Since many of the reported KDPG aldolase structures have a small molecule at the active site, such as a substrate [4], a sulfate ion [5, 12], a citrate ion [14], or a phosphate ion [5], it is not easy to determine the position of catalytic water molecules before the substrate binds to the active site. Furthermore, the absence of the structure in complex with KDPG or a substrate/product at the P-loop limits our understanding of how the substrate/ product interacts with the enzyme in this region.

KDPG aldolases from E. coli, Pseudomonas putida, and Z. mobilis have been reported to possess broad substrate specificity with regard to the electrophilic component [15]. The E. coli and P. putida aldolases prefer the D-stereochemical configuration at the $\mathrm{C} 2$ position of the substrate, whereas the Z. mobilis KDPG (ZmKDPG) aldolase displays no stereochemical discrimination at the same carbon position with regard to the electrophilic substrate. Although the ZmKDPG structure has been publicly released as a sulfate-bound form (PDB ID 4BK9), its functional relationship with the revealed structure has not been described. To gain a better understanding of the substrate-binding conformation at the P-loop and the broad substrate spectrum of the ZmKDPG aldolase, we determined its crystal structure as an apo-form at a resolution of $1.8 \AA$. We also report the structure in complex with 3-phosphoglycerate (3PG) at a resolution of $2.0 \AA$; it has a sulfate ion at the catalytic site, 3PG at the P-loop, and an extra electron density near the P-loop.

\section{Materials and Methods}

\section{Production of ZmKDPG Aldolase}

The method used to prepare protein for structural studies of ZmKDPG aldolase (ZMO0997, Met1 Ala208) has been described in detail elsewhere [16]. Briefly, the full-length ZmKDPG aldolase-producing recombinant plasmid with an N-terminal $6 \times$ His fusion tag was transformed into E. coli BL21 Star (DE3), and the expressed recombinant protein was purified by sequential chromatographic steps of Ni-ion affinity and ion exchange.

\section{Crystallization, Data Collection, and Structure Determination}

For crystallization, the purified ZmKDPG aldolase was concentrated to $8.2 \mathrm{mg} / \mathrm{ml}$ in a buffer consisting of $20 \mathrm{mM}$ Tris$\mathrm{HCl}(\mathrm{pH} 7.5)$ and $300 \mathrm{mM} \mathrm{NaCl}$. The protein concentration was determined using an extinction coefficient of $0.331 \mathrm{mg} \cdot \mathrm{ml}^{-1} \cdot \mathrm{cm}^{-1}$ at $280 \mathrm{~nm}$, which was calculated from its amino acid sequence. Attempts to crystallize ZmKDPG aldolase were performed at $295 \mathrm{~K}$ using the hanging-drop vapor-diffusion method. Suitable crystals for diffraction experiments were obtained from a precipitant solution containing $25 \%(\mathrm{w} / \mathrm{v})$ polyethylene glycol 8,000 and $0.1 \mathrm{M}$ Bis-Tris ( $\mathrm{pH}$ 5.5) for the apo-form, and $1.6 \mathrm{M}$ ammonium sulfate, $0.1 \mathrm{M}$ CAPS ( $\mathrm{pH} 10.5$ ), and $0.2 \mathrm{M}$ lithium sulfate for the 3PG-bound form. For diffraction experiments, crystals in the drops were immediately placed in a $100 \mathrm{~K}$ nitrogen-gas stream. The diffraction data for the apo-ZmKDPG aldolase crystal were collected at the 7A beamline of the Pohang Accelerator Laboratory (PAL) in Korea at a wavelength of $1.00 \AA$ with a per-frame oscillation of $1^{\circ}$ and a per-frame exposure of $1 \mathrm{sec}$. A total of 360 images were collected on the ADSC-Q270 CCD detector. Indexing, integration, and scaling of the reflections were performed using the HKL2000 suite [17]. The electron density was calculated from molecular replacement using the PHENIX program [18]. Further model building was performed manually with WinCoot [19], and subsequent refinement was performed with PHENIX [18]. The data and refinement statistics are summarized in Table 1. To get the 3PG-bound structure, the crystal were soaked for $1 \mathrm{~h}$ in a precipitant solution containing $10 \mathrm{mM}$ 3PG. Diffraction data were also collected at the 7A beamline of PAL by applying the same parameters as those for the apo-form.

\section{Accession Numbers}

Coordinates and structure factors have been deposited in the Protein Data Bank with the accession numbers 5XSE and 5XSF.

\section{Results and Discussion}

\section{ZmKDPG Aldolase Has a Conserved TIM Barrel Structure}

The crystal structures for ZmKDPG aldolase were solved by a molecular replacement method, using the E. coli KDPG aldolase [6] as a search model, which has a sequence identity of $\sim 50 \%$ with the ZmKDPG aldolase. Molecular replacement indicated the presence of three protein molecules in the asymmetric unit of the orthorhombic crystal system (apo-structure), which was refined to a resolution of $1.8 \AA$. The 3PG-bound structure in the cubic crystal system was also solved by molecular replacement and refined to a resolution of $2.0 \AA$ (Table 1). The data and 
Table 1. Data collection and structure refinement statistics.

\begin{tabular}{|c|c|c|}
\hline Data collection & Apo-structure & 3PG-bound structure \\
\hline Space group & $\mathrm{P} 2_{1} 2_{1} 2_{1}$ & $\mathrm{~F} 4_{1} 32$ \\
\hline \multicolumn{3}{|l|}{ Unit cell dimensions } \\
\hline$a, b, c(\AA)$ & $63.70,83.02,117.21$ & $186.30,186.30,186.30$, \\
\hline$\alpha, \beta, \gamma\left(^{\circ}\right)$ & $90,90,90$ & $90,90,90$ \\
\hline Wavelength $(\AA)$ & 1.00 & 1.00 \\
\hline Resolution $(\AA)$ & $50.0-1.8(1.86-1.8)^{a}$ & $50.0-1.96(2.07-1.96)^{a}$ \\
\hline Rsym & $7.7(43.0)$ & $6.8(39.9)$ \\
\hline Rpim & $3.9(25.3)$ & $1.7(11.8)$ \\
\hline$I / \sigma(I)$ & $17.0(2.2)$ & $15.9(3.4)$ \\
\hline Completeness (\%) & $98.5(95.8)$ & $100.0(100.0)$ \\
\hline Redundancy & $8.2(4.6)$ & $27.9(18.3)$ \\
\hline \multicolumn{3}{|c|}{ Refinement } \\
\hline Resolution $(\AA)$ & $35.4-1.8(1.83-1.8)$ & $35.9-1.96(2.08-1.96)$ \\
\hline No. of reflections & 57,557 & 20,372 \\
\hline$R_{\text {work }} / R_{\text {free }}$ & $18.9(34.5) / 22.7(37.3)$ & $19.1(24.7) / 21.2(27.3)$ \\
\hline \multicolumn{3}{|l|}{ No. atoms } \\
\hline protein/water & $4,522 / 665$ & $1,560 / 150$ \\
\hline \multicolumn{3}{|l|}{ RMSD } \\
\hline bond lengths $(\AA) /$ angles $\left({ }^{\circ}\right)$ & $0.005 / 0.72$ & $0.006 / 0.66$ \\
\hline \multicolumn{3}{|l|}{ Average B-values $\left(\AA^{2}\right)$} \\
\hline protein/water & $22.1 / 39.7$ & $19.4 / 34.2$ \\
\hline \multicolumn{3}{|l|}{ Ramachandran plot (\%) } \\
\hline favored/allowed/outliers & $98.4 / 1.6 / 0$ & $99.0 / 1.0 / 0$ \\
\hline
\end{tabular}

${ }^{\mathrm{a}}$ The numbers in parentheses are the statistics from the highest resolution shell.

refinement statistics are summarized in Table 1 . The quality of the model was analyzed using WinCoot [19] and MolProbity [20].

The monomeric ZmKDPG protein comprises a single globular domain with nine $\alpha$-helices and eight $\beta$-strands (Fig. 1). The eight $\beta$-strands form a $\beta$-barrel in the core with two open ends in opposite directions (Fig. 1B): N- and Cterminal ends of the $\beta$-barrel. This $\beta$-barrel is surrounded by eight $\alpha$-helices $(\alpha 2-\alpha 9)$, forming a protein fold commonly called a TIM barrel structure. Whereas the N-terminal end of the $\beta$-barrel is blocked by the $\alpha 1$-helix, the $\mathrm{C}$-terminal end is exposed to the solvent-accessible region.

Likely in other KDPG aldolases [4, 12], three molecules in the apo-structure assemble to form a trimeric structure with the $\alpha 7$-helix of one protomer partially overhung on the open C-terminal end of the $\beta$-barrel of the neighboring protomer (Fig. 1B). The active site of each protomer is located in the internal cavity of the open C-terminal end of the central $\beta$-barrel, and the P-loop is $\sim 10 \AA$ apart from the catalytic site. Whereas a trimeric structure exists in the apoform, a single protein molecule is observed in the asymmetric unit of the 3PG-bound form, whose trimeric structure can be assembled among the symmetrically related molecules.

\section{ZmKDPG Aldolase Has a Conserved Catalytic Site}

A lysine residue at the active sites of the KDPG aldolases forms a Schiff-base intermediate with the C2-atom of the substrate, and a glutamate residue acts as a general acid/ base $[4,12]$. The formation of carbinolamine during catalysis was attributed to a discontinuity of the electron density for the side chain of the catalytic lysine residue [4, 12, 21].

The spatial position of the bound sulfate ion in the $\beta$-barrel of ZmKDPG coincides with those of the carboxylate group of the pyruvate found in the catalytic sites of the E. coli [4] and T. maritima [12] KDPG aldolase structures. Hence, the sulfate ion observed within the $\beta$-barrel of the 3PG-bound structure clearly indicates the active site of the ZmKDPG aldolase, where the two conserved residues (K128 and E41) interact with the sulfate ion (Fig. 2). The Zymomonas KDPG catalytic site is similar to those of E. coli and Thermotoga KDPG aldolases with most of the residues at the structurally equivalent positions. Interestingly, the electron density for the side chain of the catalytic K128 is 
A

Zymomonas
Thermotoga
Haemophilus
Pseudomonas
Oleispira
Escherichia

Zymomonas Thermotoga Haemophilus Pseudomonas Oleispira

Zymomonas
Thermotoqa
Haemophilus
Pseudomonas
Oleispira
Escherichia

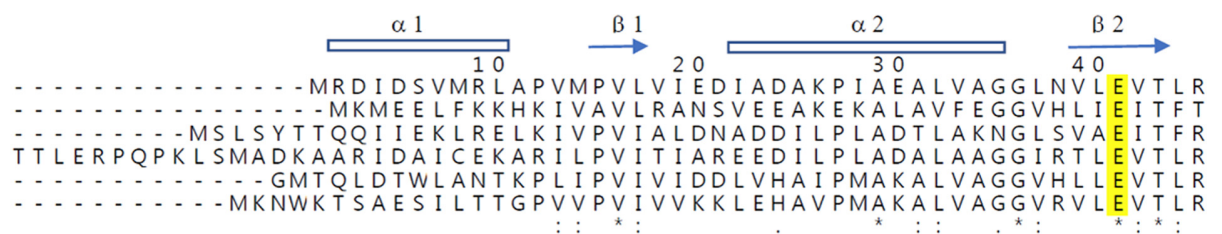

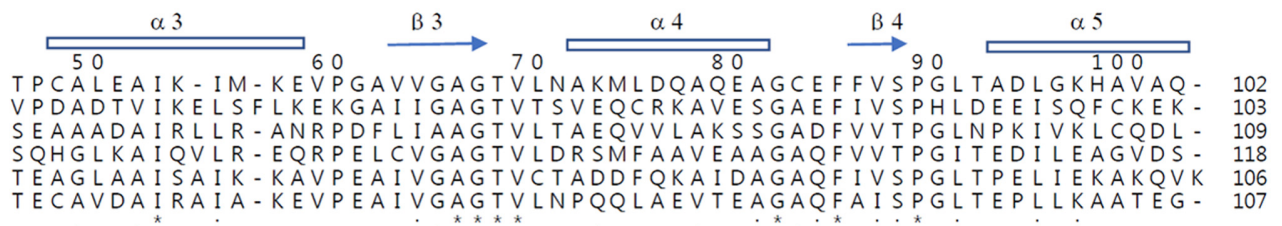

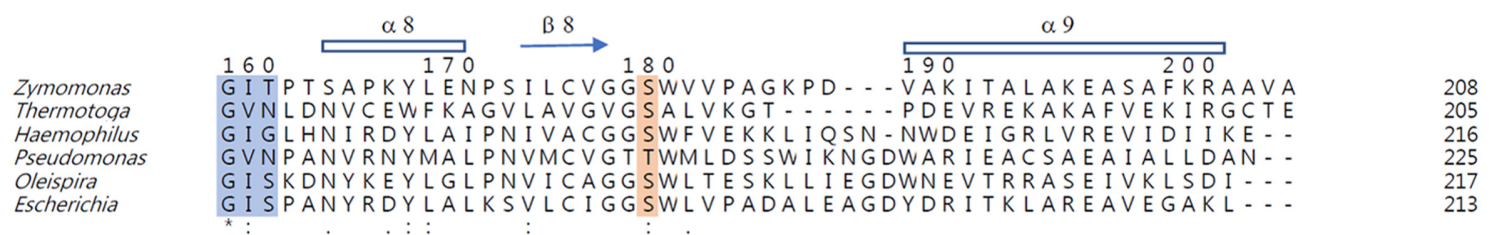

B

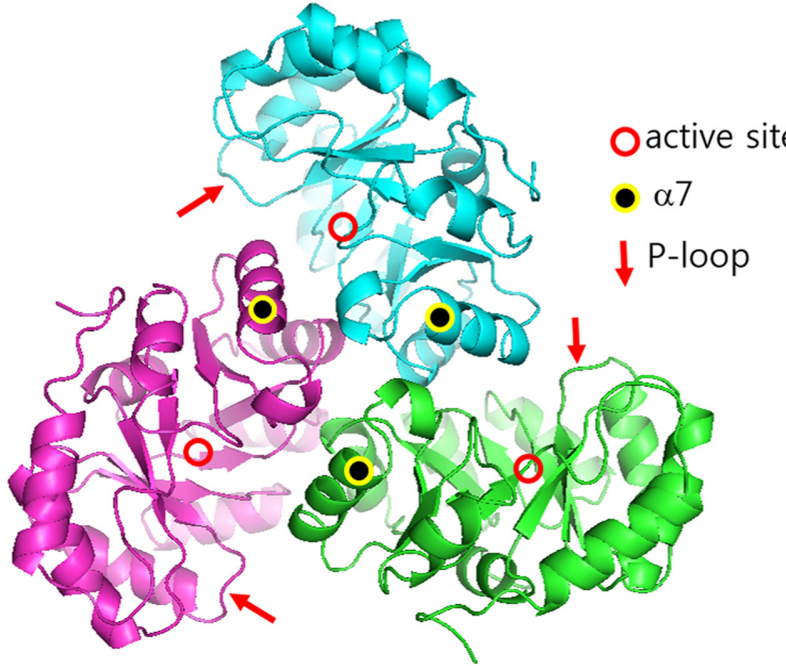

C

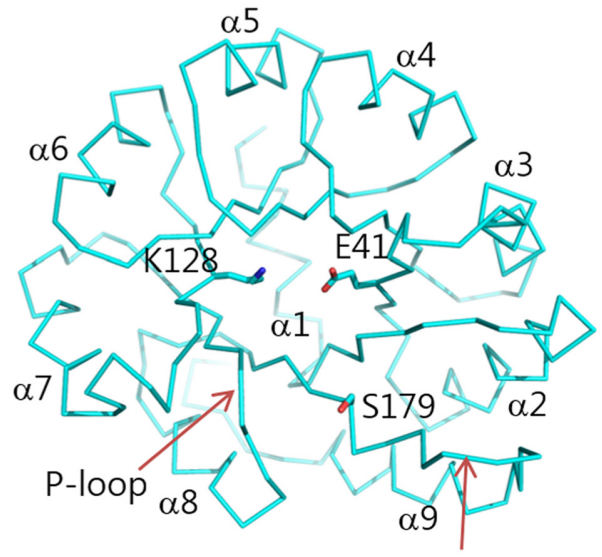

$\beta 8-\alpha 9$ loop

Fig. 1. Structural features of Zymomonas 2-keto-3-deoxy-6-phosphogluconate (ZmKDPG) aldolase.

(A) Sequence alignment of ZmKDPG aldolase with other related proteins. Sequence alignment was performed using Clustal Omega from the European Bioinformatics Institute. Identical residues are marked with an asterisk "*", and similar residues are marked with ":" and "..", respectively. The $\alpha$-helices (rectangles) and $\beta$-strands (arrows) are numbered in order of their appearance in the ZmKDPG aldolase structure. The catalytic residues (E41 and K128), the phenylalanine residue (F130), the P-loop (T156-T160), and the phosphate-interacting serine/threonine residue (S179) are indicated on yellow, grey, blue, and orange backgrounds, respectively. Abbreviations: Zymomonas, KDPG aldolase from Z. mobilis ZM4; Thermotoga, KDPG aldolase from T. maritima; Haemophilus, KDPG aldolase from H. influenzae; Pseudomonas, KDPG aldolase from P. putida; Oleispira, KDPG aldolase from O. antarctica; Escherichia, KDPG aldolase from E. coli. (B) A cartoon diagram of the revealed trimeric ZmKDPG aldolase structure. The three ZmKDPG aldolase protomers in the asymmetric unit of the apo-structure are differentiated by color. The active sites and three $\alpha 7$-helices in the 3-fold axis are indicated. (C) A ribbon diagram of a monomeric ZmKDPG aldolase. Key residues (E41 and K128) at the catalytic site and the serine residue (S179) near the P-loop are displayed with stick models. Two significant loops near the active site are indicated. All figures, except for Fig. 1A, were prepared by the PyMol Molecular graphics program by Schrödinger, LLC. 
A
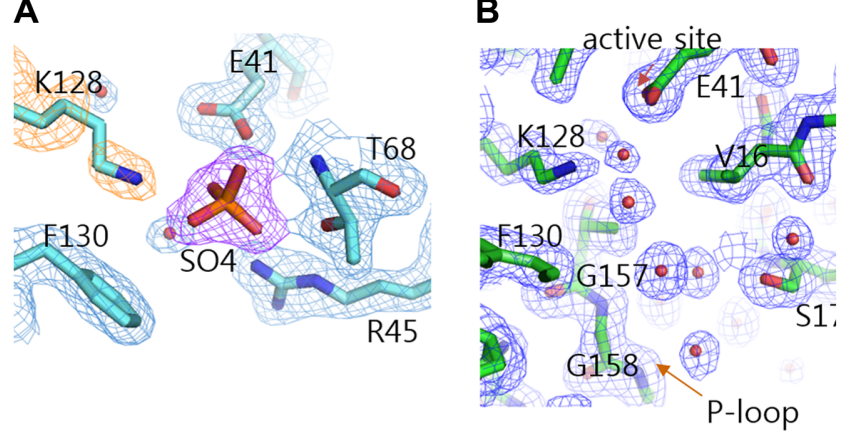

C

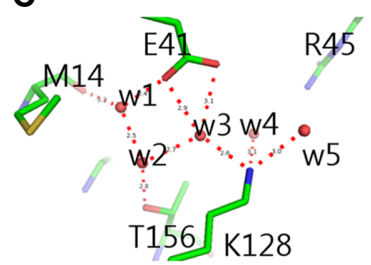

E
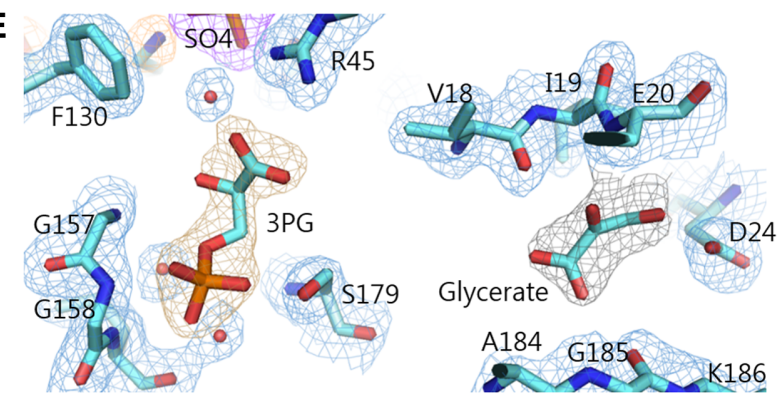

Fig. 2. Active site of the Zymomonas 2-keto-3-deoxy-6phosphogluconate aldolase.

(A, B) Active site of the 3-phosphoglycerate (3PG)-bound structure (A) and the apo-structure (B). Water molecules are indicated with red spheres in the 2Fo-Fc map contoured at $1.0 \sigma$ level, and residues and the bound sulfate ion at the catalytic site are displayed with stick models. (C, D) Water molecules at the active site. Polar interactions are indicated with red-dotted lines for the apo-structure $(\mathrm{C})$ and the 3PG-bound structure (D). (E) The 3PG at the P-loop and the nearby extra electron density. Water molecules and some residues are displayed with red spheres and stick models in the 2Fo-Fc map (blue), respectively. The bound 3PG is also displayed with a stick model in the 2Fo-Fc map contoured at $1.0 \sigma$ level (orange), and the extra electron density near the P-loop (Glycerate) is displayed with a grey color.

discontinuous (Fig. 2A), like the catalytic lysine in the E. coli KDPG aldolase that forms a complex with pyruvate [4]. Since the side chain of the catalytic lysine has a defined electron density in the inorganic sulfate-bound structure of E. coli [4], the interpretation of this disrupted electron density in the ZmKDPG aldolase structure is not clear.
In the apo-structure of the ZmKDPG aldolase, several water molecules lie in the space between the P-loop and the catalytic site (Fig. 2B). Water molecules at the catalytic site form an elaborate polar interaction with protein atoms such as K128, R45, E41, and T156 (Fig. 2C), suggesting water positioning before substrate binding occurs to the catalytic site. Binding of a sulfate ion at the active site replaces the water molecules observed in the apo-structure (Fig. 2D).

\section{PG Binds to the P-Loop}

The P-loop of the KDPG aldolases has two conserved consecutive glycine residues and interacts with an inorganic sulfate or phosphate ion via peptidyl nitrogen atoms of the glycine residues (Figs. $1 \mathrm{~A}$ and 2E) [5, 12]. A conserved serine residue further interacts with the sulfate or phosphate ion at the opposite side of these two glycine residues (Fig. 2E). Mutational studies of this serine residue in the E. coli aldolase confirmed that it is related to recognition of diverse substrates [22].

In the 3PG-bound ZmKDPG structure, the 3PG molecule is observed in the space between the P-loop ( $\beta 7-\alpha 8$ loop) and the conserved S179 residue (Fig. 2E). The phosphate moiety of 3PG interacts with the P-loop and the re-oriented side chain of S179. In addition, the carboxylate moiety of 3PG is within the distance feasible for an ionic interaction with the side chain of R45.

The 3PG-complexed ZmKDPG structure clearly suggests how the P-loop and the nearby conserved serine residue simultaneously contribute to substrate binding, which provides a clue for explaining the difference in the substrate specificity of E. coli KDPG aldolase when this conserved serine is replaced with other amino acids [22].

\section{The $\beta 8-\alpha 9$ Regions of KDPG Aldolases Are Diverse and Flexible}

To date, six trimeric KDPG aldolase structures, including the ZmKDPG structure, have been reported from diverse organisms [4-6, 12, 23, 24]. Their three-dimensional structures are very similar, as indicated by the small root-meansquare deviation values among the compared monomeric C $\alpha$ atoms $(<1.5 \AA)$.

Upon superposition of their structures, the largest deviation is found in the $\beta 8-\alpha 9$ region near the P-loop (Fig. 3A), which has no sequence conservation (Fig. 1A). The corresponding regions of the compared KDPG aldolases, except for ZmKDPG aldolase with an extended loop, have an $\alpha$-helix positioned between the $\beta 1-\alpha 2$ loop and the $\alpha 9$-helix (Fig. 3B). An extra electron density is found in the space between the $\beta 8-\alpha 9$ loop and the $\beta 1-\alpha 2$ 
A

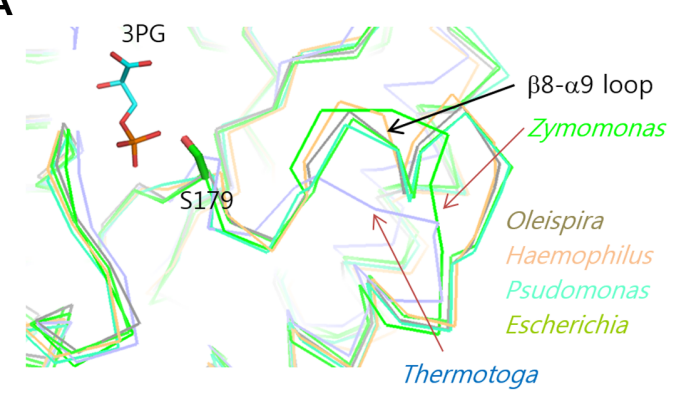

B
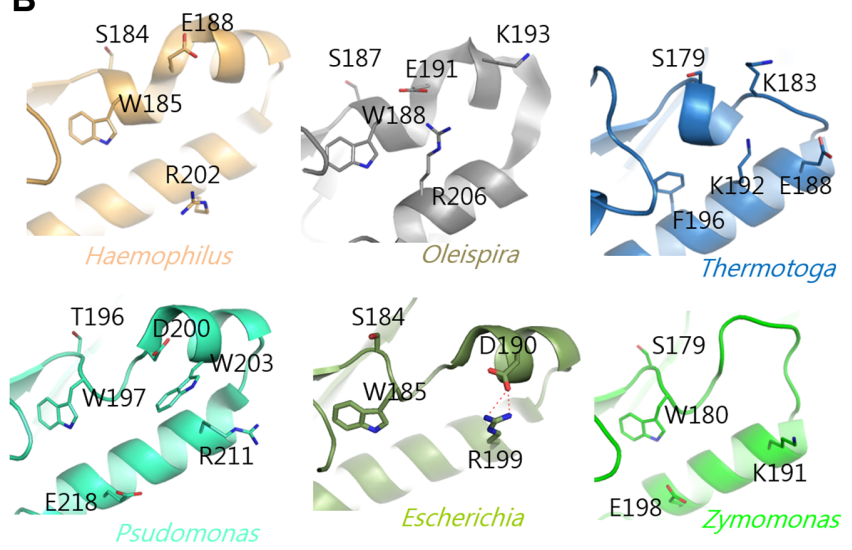

Fig. 3. Structural diversity of the $\beta 8-\alpha 9$ loop.

(A) Superposition of the six compared KDPG aldolases. Six KDPG aldolase structures (displayed with different $\mathrm{C} \alpha$ tracings) were superposed and the $\beta 8-\alpha 9$ loop-containing regions were magnified. The 3-phosphoglycerate (3PG) molecule at the P-loop and the side-chain for the nearby S179 of the Zymomonas KDPG aldolase are displayed with stick models. (B) Ribbon diagrams of the compared $\beta 8-\alpha 9$ loops in KDPG aldolases from different organisms. Some residues of the $\beta 8$ $\alpha 9$ loops and the $\alpha 9$-helix are drawn with stick models.

loop in the 3PG-bound ZmKDPG structure. A glycerate molecule fits well within this electron density, which was probably taken from the culture medium (glycerate in a grey electron density in Fig. 2E). This electron density is located within the distance required for polar interaction with the two peptidyl nitrogen atoms of I19 and E20 and with the side-chain atoms of D24. Notably, E. coli KDPG also has an acetate ion at the structurally equivalent position to this extra electron density [4].

E. coli, P. putida, and Z. mobilis KDPG aldolases can accommodate diverse substrates of the electrophilic component [15]. Furthermore, reported KDPG aldolases, including the ZmKDPG aldolase in this study, have shown common structural features, with a unique difference in the $\beta 8-\alpha 9$ region containing a phosphate-interacting serine residue near the P-loop $[4-6,12,23,24]$. Therefore, the reported substrate diversity of Zymomonas KDPG aldolase is most likely to be related to the flexibility of the $\beta 8-\alpha 9$ region. Serine residues in the KDPG aldolases of Haemophilus, Thermotoga, and Oleispira belong to the $\alpha$-helix (Fig. 3B), which confers limited spatial rotameric positions to this conserved serine residue. On the other hand, the conserved serine residues of Escherichia and Zymomonas aldolases and the threonine residue of Pseudomonas aldolase belong to the loops, implying their broader conformations in comparison with other KDPG aldolases. It is worthwhile to recall that E. coli KDPG aldolase could recognize large hydrophobic substrates when this conserved serine was varied [22], indicating that it can have various conformations depending on the substrate. In this context, the absence of the $\alpha$-helical structure in the $\beta 8-\alpha 9$ region of ZmKDPG aldolase might confer higher flexibility to this serinecontaining loop than the $\beta 8-\alpha 9$ regions of $E$. coli and Pseudomonas aldolases. Notably, ZmKDPG aldolase has shown a higher catalytic activity than Escherichia and Pseudomonas aldolases on the aldol condensation reaction with bulkier substrates such as D-ribose5-phosphate and 2furaldehyde [15].

\section{Concluding Remarks}

We have shown that the 3PG-bound structure suggests how the substrate can bind to the P-loop. The overall structural features reported in other KDPG aldolases are also conserved in the ZmKDPG aldolase, indicating that it shares a common enzymatic mechanism to other class I KDPG aldolases. A unique structural divergence among the six compared KDPG aldolases was observed at one region near the P-loop (Fig. 3). Since ZmKDPG aldolase has not shown a noticeable conformational change upon binding of 3PG, the flexible $\beta 8-\alpha 9$ loop of ZmKDPG aldolase is probably related to the broad substrate spectrum of the enzyme reported in the previous study [15].

Unlike KDPG aldolases from E. coli and P. putida that prefer substrates with a D-stereochemical configuration at the C2 atom, the ZmKDPG aldolase displayed no stereochemical discrimination of the electrophilic substrate at the same atom [15]. Residues at the catalytic site, the Ploop, and the conserved phenylalanine residue (F130) that was suggested to confer the stereoselectivity at the C2 atom [4] show no variation in their spatial positions. Therefore, it is not clear, from a structural perspective, why the stereochemistry for the $\mathrm{C} 2$ atom of the substrate by the ZmKDPG aldolase is distinct from that of E. coli and of P. putida, indicating the necessity for further structural and biochemical characterizations of this enzyme. 


\section{Acknowledgments}

The X-ray diffraction experiments used Beamline 7A at the Pohang Accelerator in Korea. This work was supported by Basic Science Research (NRF Grant No. 2014R1A2A2A01004915 and 2017R1D1A3B03032278) of the National Research Foundation (NRF) of Korea funded by the Ministry of Education, Science and Technology (MEST) of Korea. We thank Dr. S.-C. Ha, Pohang Light Source, Korea, for helping with data collection.

\section{Conflict of Interest}

The authors have no financial conflicts of interest to declare.

\section{References}

1. Romano AH, Conway T. 1996. Evolution of carbohydrate metabolic pathways. Res. Microbiol. 147: 448-455.

2. Seo JS, Chong H, Park HS, Yoon KO, Jung C, Kim JJ, et al. 2005. The genome sequence of the ethanologenic bacterium Zymomonas mobilis ZM4. Nat. Biotechnol. 23: 63-68.

3. Conway T, Fliege R, Jones-Kilpatrick D, Liu J, Barnell WO, Egan SE. 1991. Cloning, characterization, and expression of the Zymomonas mobilis eda gene that encodes 2-keto-3-deoxy6-phosphogluconate aldolase of the Entner-Doudoroff pathway. Mol. Microbiol. 5: 2901-2911.

4. Allard J, Grochulski P, Sygusch J. 2001. Covalent intermediate trapped in 2-keto-3-deoxy-6-phosphogluconate (KDPG) aldolase structure at $1.95-\AA$ resolution. Proc. Natl. Acad. Sci. USA 98: 3679-3684.

5. Bell BJ, Watanabe L, Rios-Steiner JL. 2003. Structure of 2keto-3-deoxy-6-phosphogluconate (KDPG) aldolase from Pseudomonas putida. Acta Crystallogr. D Biol. Crystallogr. 59: 1454-1458.

6. Wymer N, Buchanan LV, Henderson D, Mehta N, Botting $\mathrm{CH}$, Pocivavsek L, et al. 2001. Directed evolution of a new catalytic site in 2-keto-3-deoxy-6-phosphogluconate aldolase from Escherichia coli. Structure 9: 1-9.

7. Cooper SJ, Leonard GA, McSweeney SM, Thompson AW, Naismith JH, Qamar S, et al. 1996. The crystal structure of a class II fructose-1,6-bisphosphate aldolase shows a novel binuclear metal-binding active site embedded in a familiar fold. Structure 4: 1303-1315.

8. Joerger AC, Mueller-Dieckmann C, Schulz GE. 2000. Catalytic action of fuculose 1-phosphate aldolase (class II) as derived from structure-directed mutagenesis. J. Mol. Biol. 303: 531-543.

9. Kroemer M, Merkel I, Schulz GE. 2003. Structure and catalytic mechanism of L-rhamnulose-1-phosphate aldolase. Biochemistry 42: 10560-10568.

10. Plater AR, Zgiby SM, Thomson GJ, Qamar S, Wharton CW,
Berry A. 1999. Conserved residues in the mechanism of the E. coli class II FBP-aldolase. J. Mol. Biol. 285: 843-855.

11. Henderson DP, Shelton MC, Cotterill IC, Toone EJ. 1997. Stereospecific preparation of the N-terminal amino acid moiety of nikkomycins $K(X)$ and $K(Z)$ via a multiple enzyme synthesis. J. Org. Chem. 62: 7910-7911.

12. Fullerton SW, Griffiths JS, Merkel AB, Cheriyan M, Wymer NJ, Hutchins MJ, et al. 2006. Mechanism of the Class I KDPG aldolase. Bioorg. Med. Chem. 14: 3002-3010.

13. Mavridis IM, Hatada MH, Tulinsky A, Lebioda L. 1982. Structure of 2-keto-3-deoxy-6-phosphogluconate aldolase at 2.8 A resolution. J. Mol. Biol. 162: 419-44.

14. Wymer N, Buchanan LV, Henderson D, Mehta N, Botting $\mathrm{CH}$, Pocivavsek L, et al. 2001. Directed evolution of a new catalytic site in 2-keto-3-deoxy-6-phosphogluconate aldolase from Escherichia coli. Structure 9: 1-9.

15. Shelton MC, Cotterill IC, Novak ST, Poonawala RM, Sudarshan S, Toone EJ. 1996. 2-Keto-3-deoxy-6-phosphogluconate aldolases as catalysts for stereocontrolled carbon-carbon bond formation. J. Am. Chem. Soc. 118: 2117-2125.

16. Ryu HC, Park SY, Kim JS. 2010. Cloning, expression, purification, crystallization and preliminary $\mathrm{X}$-ray diffraction analysis of 2-keto-3-deoxy-6-phosphogluconate aldolase from Zymomonas mobilis ZM4. Acta Crystallogr. F Struct. Biol. Cryst. Comm. 66: 471-473.

17. Otwinowski Z, Minor W. 1997. Procession of x-ray diffraction data collected in oscillation mode. Methods Enzymol. 276: 307-326.

18. Adams PD, Afonine PV, Bunkoczi G, Chen VB, Davis IW, Echols N, et al. 2010. PHENIX: a comprehensive Pythonbased system for macromolecular structure solution. Acta Crystallogr. D Biol. Crystallogr. 66: 213-221.

19. Emsley P, Cowtan K. 2004. Coot: model-building tools for molecular graphics. Acta Crystallogr. D Biol. Crystallogr. 60: 2126-2132.

20. Davis IW, Leaver-Fay A, Chen VB, Block JN, Kapral GJ, Wang X, et al. 2007. MolProbity: all-atom contacts and structure validation for proteins and nucleic acids. Nucleic Acids Res. 35: W375-W383.

21. Meloche HP, Glusker JP. 1973. Aldolase catalysis: single base-mediated proton activation. Science 181: 350-352.

22. Cheriyan M, Toone EJ, Fierke CA. 2007. Mutagenesis of the phosphate-binding pocket of KDPG aldolase enhances selectivity for hydrophobic substrates. Protein Sci. 16: 2368-2377.

23. Badger J, Sauder JM, Adams JM, Antonysamy S, Bain K, Bergseid MG, et al. 2005. Structural analysis of a set of proteins resulting from a bacterial genomics project. Proteins 60: 787-796.

24. Kube M, Chernikova TN, Al-Ramahi Y, Beloqui A, LopezCortez N, Guazzaroni ME, et al. 2013. Genome sequence and functional genomic analysis of the oil-degrading bacterium Oleispira antarctica. Nat. Commun. 4: 2156. 